\title{
Targeted Accrual
}

National Cancer Institute

\section{Source}

National Cancer Institute. Targeted Accrual. NCI Thesaurus. Code C71461.

The final sample size that must be attained according to the study protocol. 